preterm. Number of grandchildren increased with increasing number of children in both sexes, providing no evidence for a trade-off between quantity of offspring and their subsequent reproductive "quality".

Conclusions: Early life characteristics can affect reproductive success even in post-demographic transition populations. These effects operate via multiple pathways and include "biological" characteristics such as birthweight having an effect via social facts such as adult marital status. These findings can inform analyses of reproductive career as a determinant of health in later life in this and other similar populations. They also generate hypotheses regarding the potential long term consequences of adverse early environments in concurrent cohorts around the world.

\section{TRENDS IN MATERNAL OBESITY AND HEALTH INEQUALITIES IN A NATIONALLY REPRESENTATIVE SAMPLE OF 619323 BIRTHS IN ENGLAND, UK, 1989-2007}

${ }^{1} \mathrm{~N}$ Heslehurst, ${ }^{2} \mathrm{~J}$ Rankin, ${ }^{3} \mathrm{~J}$ Wikinson, ${ }^{4} \mathrm{CD}$ Summerbell. ${ }^{1}$ Health and Social Care Research Institute, University of Teesside, Middlesbrough, Tees Valley, UK; ${ }^{2}$ Institute of Health and Society, Newcastle University, Newcastle upon Tyne, UK: ${ }^{3}$ North East Public Health Observatory, Durham University, Stockton, UK; ${ }^{4}$ School of Medicine and Health, Durham University, Stockton, UK

\section{doi:10.1136/jech.2009.096701f}

Objectives: Maternal obesity has serious implications to the health of both mothers and infants, including maternal and neonatal death, stillbirth, congenital anomalies, poor breastfeeding rates, and obesity in the offspring. There are also additional complications during antenatal, intrapartum, and postnatal periods which impact on maternity services. However, there is an absence of national statistics for maternal obesity in the UK. This study is the first to describe a nationally representative maternal obesity research dataset in England.

Design of the Study: Descriptive epidemiological study using routinely collected data.

Setting: 34 maternity units in England.

Participants: 619323 women who delivered at the maternity units sampled, between January 1989 and December 2007.

Main Outcome Measures: Trends in first trimester maternal BMI status over time and geographical distribution of maternal obesity by Government Office Regions in England. Demographics of the population were analysed to identify any maternal obesity associated health inequalities, including maternal age, parity, ethnic group, deprivation, and employment. All demographics were tested for multicollinearity. Logistic regression adjusted for all included demographics as confounders.

Results: The demographic characteristics of the study population were representative when compared to census and deprivation data. Obesity in the first trimester of pregnancy is significantly increasing over time, having more than doubled from $7.6 \%$ to $15.6 \%$ over the 19 years studied $(p<0.001)$. There is significant geographic variation in the incidence of maternal obesity, with the West Midlands, Yorkshire and the Humber and North East Government Office Regions having higher than national average incidence of first trimester obesity. There are health inequalities associated with maternal obesity, including increased odds of being obese with increasing age (1.02, 95\% CI 1.02 to 1.02$)$, parity $(1.17,95 \%$ CI 1.16 to 1.18$)$, black ethnic group $(1.78,95 \%$ CI 1.70 to 1.87$)$, and deprivation $(2.20,95 \%$ CI 2.13 to 2.28$)$. There is also an association between super morbid obesity and unemployment $(1.50,95 \% \mathrm{CI}$ 1.12 to 2.02 ).

Conclusions: The increase in maternal obesity at booking has yielded an additional 47500 women per year requiring high dependency care in England. The demographics of women most at risk of first trimester obesity highlight health inequalities associated with maternal obesity which need to be addressed.

\section{INHERITED RISK OF PRE-ECLAMPSIA: USING TWO APPROACHES FOR ANALYSIS}

S Bhattacharya, EA Raja, DM Campbell, AJ Lee. Division of Applied Medicine, University of Aberdeen, Aberdeen, UK

doi:10.1136/jech.2009.096701g

Background: Several previous research reports have suggested a genetic predisposition to pre-eclampsia but none have demonstrated the effect separately in nulliparous and parous women in the context of other risk or protective factors.

Objective: To assess the magnitude of genetic predisposition to pre-eclampsia with reference to other risk factors in nulliparous and parous women.

Material and Method: The Aberdeen Maternity and Neonatal Databank records all pregnancy and delivery details occurring in Aberdeen, Scotland since 1950. It has now become possible to link pregnancy records of mothers and grandmothers to those of the daughters. Using a nested case control design within this intergenerational cohort, statistical modelling was done with known risk/protective factors for pre-eclampsia, separately for nulliparous and parous women. Conditional logistic regression was used to compare characteristics between parous pre-eclamptics and year and parity matched normotensive controls. In a separate analysis, including all parous women, we used a multilevel approach based on Generalised Estimating Equation (GEE) and specified the link function as binomial. We assumed a working exchangeable correlation of having preeclampsia within a daughter in her pregnancies. Odds ratios (OR) and 95\% CI were estimated through GEE with the use of robust standard errors.

Results: There were 34970 mother-daughter pairs. Of the daughters, there were 1248 nulliparous and 448 parous preeclamptics. For nulliparous women, the risk factors remaining in the stepwise model were mother's history of pre-eclampsia (OR $2.13,95 \%$ CI 1.57 to 2.89 ), booking BMI $>30 \mathrm{~kg} / \mathrm{m}^{2}$ (OR 2.06, $95 \%$ CI 1.68 to 2.52), age, gestation period, and booking diastolic blood pressure. Smoking $\sim 10$ cigarettes a day was protective against preeclampsia (OR 0.52 , 95\% CI 0.44 to 0.62 ). For multiparae, the risk factors included pre-eclampsia in the initial pregnancy (OR 8.80, 95\% CI 1.54 to 50.23), advanced age at delivery (OR 3.09, 95\% CI 1.69 to 5.66 ) and BMI $>30 \mathrm{~kg} / \mathrm{m}^{2}$ (OR 2.61, 95\% CI 1.62 to 4.20 ). Smoking 10 or more cigarettes per day was protective (OR 0.57 , $95 \%$ CI 0.35 to 0.94 ). A history of maternal pre-eclampsia was not independently associated with an increased risk of development of pre-eclampsia in the multiparae after adjusting for other covariates. Results were similar using the GEE approach.

Conclusion: In nulliparous women, a history of maternal preeclampsia was associated with more than doubling of risk of preeclampsia. In multiparae, this association was not observed, although a history of pre-eclampsia in a previous pregnancy was strongly associated with increased risk, suggesting genetic susceptibility.

\section{BREASTFEEDING IS ASSOCIATED WITH IMPROVED CHILD COGNITIVE DEVELOPMENT: EVIDENCE FROM THE UK MILLENNIUM COHORT STUDY}

${ }^{1} \mathrm{MA}$ Quigley, ${ }^{1} \mathrm{C}$ Hockley, ${ }^{1} \mathrm{C}$ Carson, ${ }^{2} \mathrm{Y}$ Kelly, ${ }^{3} \mathrm{M}$ Renfrew, ${ }^{4} \mathrm{~A}$ Sacker. ${ }^{1}$ National Perinatal Epidemiology Unit, University of Oxford, Oxford, UK; ${ }^{2}$ Department of Epidemiology and Public Health, University College London, London, UK; ${ }^{3}$ Mother and Infant Research Unit, University of York, Heslington, York, UK; ${ }^{4}$ Institute for Social and Economic Research, University of Essex, Colchester, Essex, UK

doi:10.1136/jech.2009.096701h

Objective: To assess the relationship between breastfeeding and child cognitive development, and whether this relationship varies according to prematurity. 